\title{
Cardiopulmonary exercise testing in children and adolescents
}

\section{CARDIOLOGY \\ EXERCISE PHYSIOLOGY

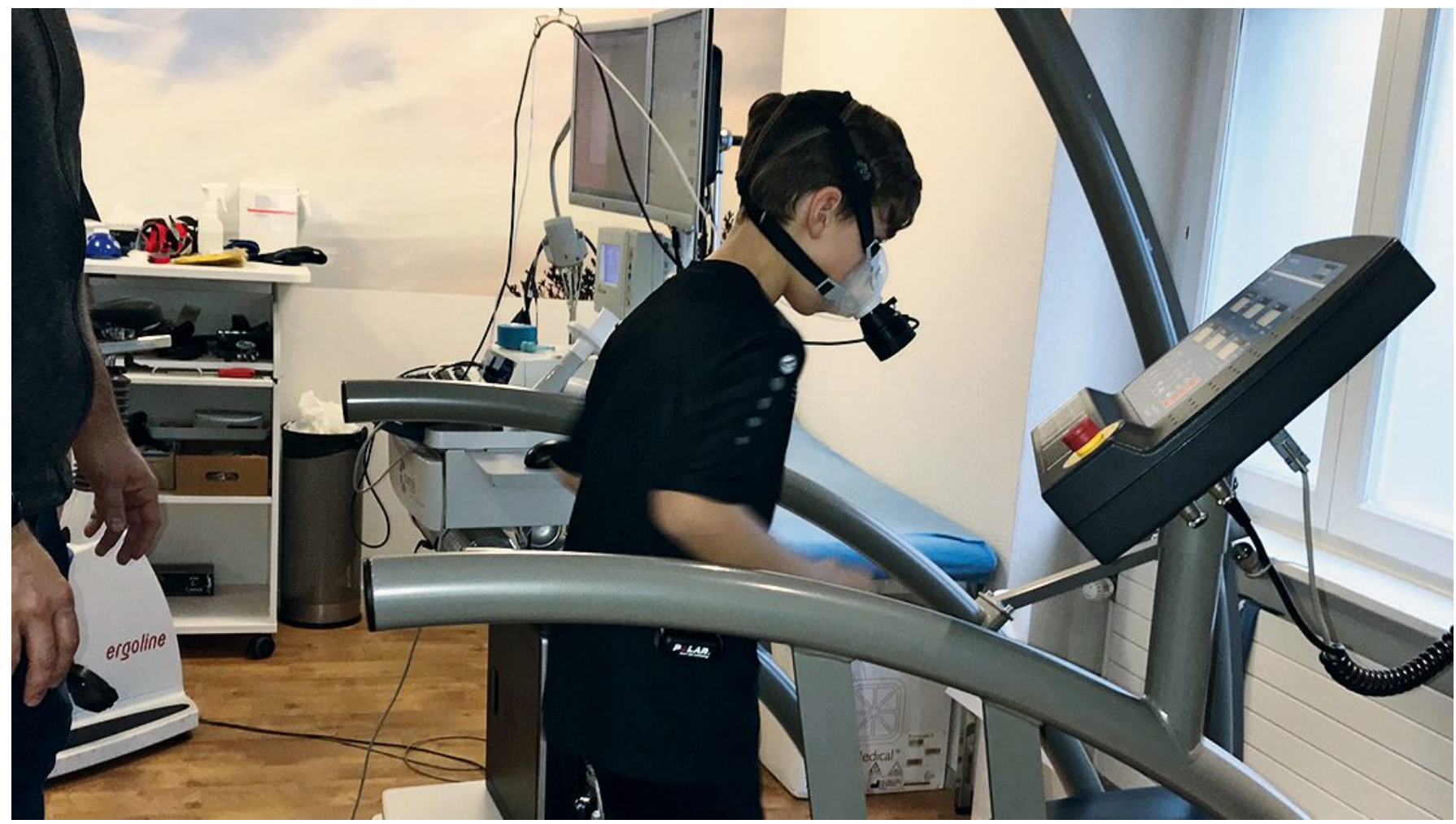

Kardiopulmonale Leistungsdiagnostik bei Kindern und Jugendlichen

\section{Stuessi $\mathrm{C}^{1}{ }^{2}$, Rinderer $\mathrm{B}^{2}$}

${ }^{1}$ SportClinic und sihldoc4kids, Zürich

${ }^{2}$ Swiss Olympic Sports Medical Base, Münsterlingen TG

\section{Abstract}

In cardiopulmonary exercise testing with children and adolescents, age specific protocols are used together with tools adjustable to their body dimension and development. Assessing weight, height und pubertal stage is a prerequisite for the interpretation of every test. Indications for exercise testing are airway symptoms and findings limited performance, chronic diseases, planning of trainings and scientific 
studies. The more tests are standardized and used on a large scale, the more normal values are available to compare individual results. However, the interindividual variability of measured values is high, depending as much from the developmental stage of the individual as from protocols, tools and the performing laboratory. Tests are mainly incremental step or ramp tests, test duration should not exceed 10-12 min.

\section{Zusammenfassung}

Für die kardiopulmonale Leistungsdiagnostik werden altersspezifische Protokolle und gemäss Wachstum und Entwicklung einstellbare Geräte eingesetzt. Gewicht, Grösse und Pubertätsstadium müssen für jeden Test erfasst und in die Auswertung mit einbezogen werden. Indikationen sind Atemwegs-Symptome beim Sport, eingeschränkte Leistungsfähigkeit, chronische Krankheiten, die Trainingssteuerung sowie wissenschaftliche Fragestellungen. Je standardisierter und je länger gebräuchlich die Tests sind, desto mehr Normwerte stehen zum Resultatvergleich zur Verfügung. Die interindividuelle Variabilität der Messwerte ist allerdings erheblich und hängt neben dem Entwicklungsstand auch wesentlich von den verwendeten Protokollen und Geräten und dem Verfahren im jeweiligen Labor ab. Meist werden Stufenoder Rampenprotokolle verwendet, die Testdauer soll nicht mehr als 10-12 min betragen.

\section{Einleitung}

Für die Leistungsdiagnostik bei Kindern und Jugendlichen werden Methodik und Equipment analog zu der bei Erwachsenen verwendet. Da Kinder und Jugendliche aber keine «kleinen Erwachsenen» sind, kommen altersspezifische Protokolle und Geräte zum Einsatz, und die Resultate werden altersspezifisch dargestellt und beurteilt, beispielsweise mittels Percentilen [1,2]. Dabei muss die ganze Spanne der Alters-, Grössenund Gewichtsentwicklung abgedeckt werden. Wachstum und Entwicklung verlaufen bei beiden Geschlechtern unterschiedlich und nicht linear [1] und sind mit etwa 18 Altersjahren abgeschlossen. In ihrem Ablauf bildet die Pubertät den markantesten Einflussfaktor der hormonellen Veränderungen, äusserlich sichtbar sind vor allem der Pubertätswachstumsspurt, die deutliche Veränderung der Körperzusammensetzung und die eigentliche Pubertätsentwicklung, bei der man fünf Stadien unterscheidet [3,4]. Der Pubertätsbeginn bei Mädchen liegt etwa zwei Jahre vor dem der Knaben, noch grösser sind aber die interindividuellen Unterschiede innerhalb der normalen Entwicklung, vor allem bei den Wachstumsparametern und dem Zeitpunkt des Pubertätsbeginns. So liegt die Normbreite bei der Menarche, als markanteste und am präzisesten bestimmbare Entwicklungsstufe, zwischen 11 und 15 Jahren [3]. Jugendliche Sportler, welche früh in die Pubertät eintreten («Frühentwickler»), haben in den meisten Sportarten einen biologischen Vorteil und werden häufig - und zu Unrecht - bezüglich ihres Leistungspotenzials höher eingeschätzt als ihre spät pubertierenden Alterskollegen («Spätentwickler»). Demzufolge müssen bei jedem Leistungstest neben chronologischem Alter, Gewicht und Grösse auch das biologische Alter mit dem Pubertätsstadium und zudem die Körperzusammensetzung mitberücksichtigt werden und sind Teil der Testmethodik und -Interpretation.

\section{Indikationen für Leistungsdiagnostik bei Kindern und Jugendlichen}

Die Indikationen für kardiopulmonale Leistungsdiagnostik bei Kindern und Jugendlichen kann man in drei grössere Gruppen einteilen. Die wohl häufigsten Indikationen bilden medizinische Untersuchungen, 
allen voran Pathologien aus den Bereichen «Lunge», «Herz-Kreislauf» und «Stoffwechsel». Dabei werden Kinder und Jugendliche aufgrund von Symptomen vorgestellt, die sie selbst, die Eltern, die Trainer oder das weitere Umfeld festgestellt haben und mit Begriffen umschreiben wie «Dyspnoe»/«Atemnot bei Anstrengung/Anstrengungsasthma» "ungewöhnlich hoher Puls», «erbringt nicht die erwartete Leistung/ist weniger leistungsfähig als die peers/bricht Leistung frühzeitig ab» oder «ist immer müde im Gegensatz zu früher». Weitere Indikationen sind der follow up bei chronischen Krankheiten, allen voran Lungenpathologien wie Cystische Fibrose [5,6] oder (operierte) Herzvitien [7]. Noch zu wenig oft getestet werden Diabetiker und Patienten mit Adipositas. Zu deren Therapie gehören ganz klar Sport und Bewegung, diese Patienten bekommen aber selten ein Feedback dafür, zu welchen Leistungen sie fähig sind und in welchen Belastungsbereichen sie trainieren sollen. Eine weitere Indikation bildet sodann die Leistungsdiagnostik für die Trainingssteuerung und für wissenschaftliche Untersuchungen.

\section{Testgeräte - Anpassungen an Alter und Grösse}

Als Testgeräte werden meist Laufband oder Fahrradergometer verwendet. Sie sind bewährt und sie erlauben eine präzise Steuerung der Belastung. Während Kinder ab ca. 4-5 Jahren auf dem Laufband getestet werden können, reicht die Beinkraft in diesem Alter oft für eine hohe (Aus-)Belastung auf dem Fahrradergometer noch nicht aus. Als Minimalleistung, die von jüngeren Kindern erbracht werden muss, um auf dem Fahrradergometer testen zu können, gelten 10-20 W, als minimale Körpergrösse für die Verwendung von handelsüblichen Fahrradergometern 100-120 cm. Beim Fahrradergometer muss zwingend die Einstellung sowohl der Sattelposition als auch der Kurbellänge und der Handgriff-Position an die Körpergrösse des Kindes möglich sein. Die Grösse des Einflusses der Kurbellänge wird nicht einheitlich beurteilt. Empfohlen werden $13 \mathrm{~cm}$ mit 6 Jahren, $15 \mathrm{~cm}$ mit 8-10 Jahren und $16 \mathrm{~cm}$ mit 14 Jahren [8]. Auch Ruderergometer sind für entsprechende Fragestellungen gebräuchlich, während noch sportartspezifischere Geräte wie z.B. Kanu-Ergometer für Kinder und Jugendliche kaum standardisiert sind. Für die Messung der Atmungsparameter bei einer Ergospirometrie werden weiche, oronasale Gesichts-Masken in passender Grösse und damit passendem Totraumvolumen gut toleriert, während die Messung mit Mundstück und Nasenclip von vielen Kindern und Jugendlichen als unangenehm empfunden wird und Grund zum vorzeitigen Testabbruch sein kann. 


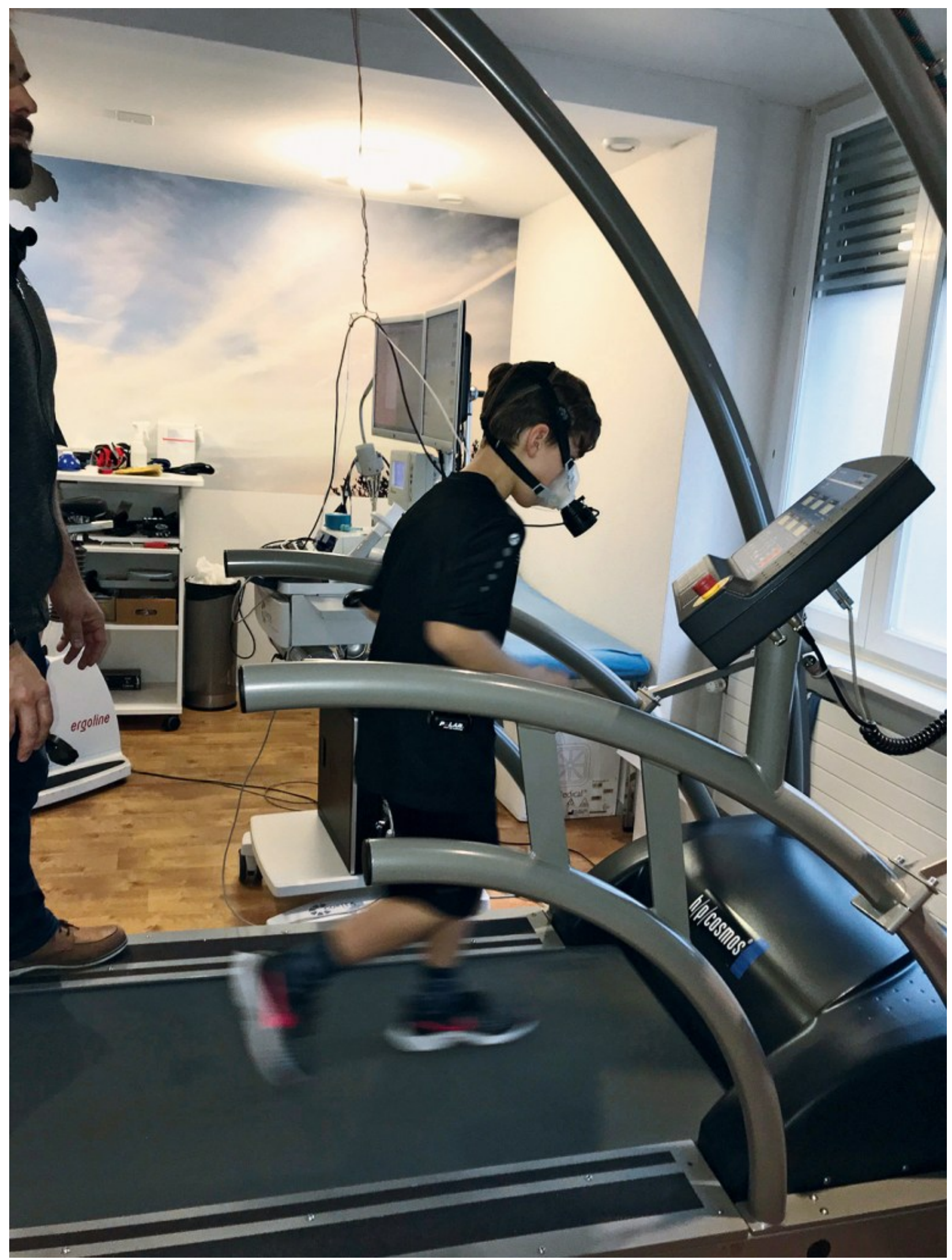




\section{Untersuchungsmethodik und Protokolle}

Am häufigsten werden - gerade bei medizinischer Indikation - Stufen- und Rampentests verwendet, also Protokolle mit ansteigender Belastung [9,10,11]. Protokolle mit hoher Standardisierung und breiter Verwendung haben eine breite Basis an Normwerten und erlauben eine bessere Vergleichbarkeit der erhobenen Daten mit der Literatur. Die gemessenen Werte unterscheiden sich jedoch je nach verwendetem Protokoll und von Labor zu Labor. Bei speziellen Fragestellungen werden seltener Protokolle mit konstanter Belastung oder konstanter Belastungszeit gewählt.

Die Beurteilung der subjektiven Belastungsintensität muss altersgerecht erfolgen. Kinder und oft auch Jugendliche verstehen die Borg 6-20 Skala nicht, eine Skala von 1-10 passt besser zu ihrem Zahlenverständnis. Speziell zu diesem Zweck wurden die OMNI RPE (Rate of Perceived Exertion) 0-10 scale oder die PCERT-Skala entwickelt [12,13,14], welche die Belastungsintensität mit Zahlenwerten 0-10, mit einer Stufendarstellung, mit Worten und mit Bildern beschreibt.

Kritische Punkte aller Protokolle sind, die Anfangsstufe und die Stufenhöhe so zu wählen, dass innert einer Testdauer von $10 \pm 2$ min ein gesetztes Belastungsziel - meist die Ausbelastung - erreicht wird. Diese Dauer wird auch von jüngeren Kindern meist ohne Motivationsverlust akzeptiert. Der Stufenanstieg soll zudem gleichförmig sein und von den Probanden gut toleriert werden. Ob auf dem Laufband 30 s-Pausen zwischen den Stufen zur Lactatgewinnung eingelegt werden oder ob eine Lactatbestimmung nur vor und nach der Belastung oder gar nicht erfolgt, richtet sich nach der Fragestellung und dem Alter der Kinder und Jugendlichen.

Laufband-Protokolle: Bewährt haben sich auch bei Kindern und Jugendlichen Protokolle, welche die Steigung fix zwischen 0-1,5\% einstellen und die Geschwindigkeit mit Inkrementen von 1-1,5 km/h erhöhen. Physiologisch nicht wirklich vergleichbar sind Stufen, welche "gehend» absolviert werden mit solchen, bei denen «gerannt» wird. Es macht somit Sinn, die Eingangsstufe so zu wählen, dass Rennen («Laufen») ab der ersten Stufe notwendig ist. Vor jedem Test sollen sich die Probanden auf einer oder zwei niedrigen Stufen einlaufen können - dies lässt gut abschätzen, welche Eingangsstufe gewählt werden soll. Eine Ausnahme davon bildet die Fragestellung nach pulmonaler Obstruktion unter Belastung, welche ohne Einlaufen provoziert werden muss.

Sehr gut bewährt hat sich die von Swiss Olympic empfohlene Untersuchung mit Steigung 1\% (die Steigung kompensiert den fehlenden Luftwiderstand) und mit Geschwindigkeitszunahme von 1,2 km/h pro Stufe [15], welche für jüngere Kinder und untrainierte Jugendliche auch auf 0,5-1 km/h gesenkt werden kann. Bei sehr leistungsfähigen Jugendlichen kann ein höherer Stufenanstieg gewählt werden. Bei unbekanntem Trainingszustand soll ein bewährtes Protokoll gewählt werden und bei Wiederholungstests über mehrere Jahre aus Gründen der Vergleichbarkeit dasselbe Protokoll gewählt werden.

Die ältesten und damit bewährtesten Laufband-Test-Protokolle wurden von Bruce und von Balke schon vor über 50 Jahren angewandt, existieren in verschiedenen Modifikationen und - ihre grössten Nachteile beginnen mit fixer Geschwindigkeit und Steigung und steigern für den Stufenanstieg entweder nur die Steigung oder Steigung und Geschwindigkeit. Viele Kinder und Jugendliche empfinden die Initialbelastung als zu gering sowie eine starke Steigung des Laufbands oder die Kombination der Erhöhung von Steigung und Geschwindigkeit als unangenehm, was zu vorzeitigem Leistungsabbruch führen kann.

Fahrradergometer-Protokolle: Zu den für Kinder und Jugendliche gut geeigneten Protokollen gehören das Giessener Protokoll [16] mit Anfangsbelastung von $1 \mathrm{~W} / \mathrm{kg}$, Stufenhöhe von $1 \mathrm{~W} / \mathrm{kg}$ und Stufendauer von 2 min, getestet an grossen Fallzahlen, sowie die von Swiss Olympic empfohlenen Protokolle [15] mit 
Anfangsbelastung von 40-100 W (=1-2 W/kg) und einer Stufenhöhe von 30 W, während die Stufendauer dem Alter und der Fragestellung angepasst werden soll

( 2 min oder $3 \mathrm{~min}$ ). Insbesondere eignet sich hier die Limitierung des Stufenanstiegs auf max. 30 W/kg; nur sehr gut leistungsfähige Jugendliche tolerieren Anfangsbelastungen von $>2 \mathrm{~W} / \mathrm{kg}$ oder Stufenhöhen von $50 \mathrm{~W}$ und mehr ohne Probleme.

Bei jüngeren Kindern oder vermutetem Trainingsmangel können auch Stufen von 0,5 W/kg KG gewählt werden, beispielsweise ab der dritten Belastungsstufe.

Freies Laufen ist deutlich weniger standardisierbar. Wenn grössere Gruppen beurteilt werden sollen oder eine Ergometrie nicht zur Verfügung steht, können sowohl gesunde Probanden wie auch Patienten mit chronischen Krankheiten mit weiteren Testverfahren wie dem 10 m oder 20 m Shuttle Run - einem Pendellauf zwischen zwei Linien - zuverlässig beurteilt werden [17]. Die gelegentlich durchgeführte Belastung in einem Treppenhaus «hinauf-hinunter» sollte nicht mehr praktiziert werden, da sie nicht standardisiert ist und es sich effektiv um eine Intervallbelastung mit kurzer Stufendauer handelt, mit der keine relevanten Fragestellungen beantwortet werden können.

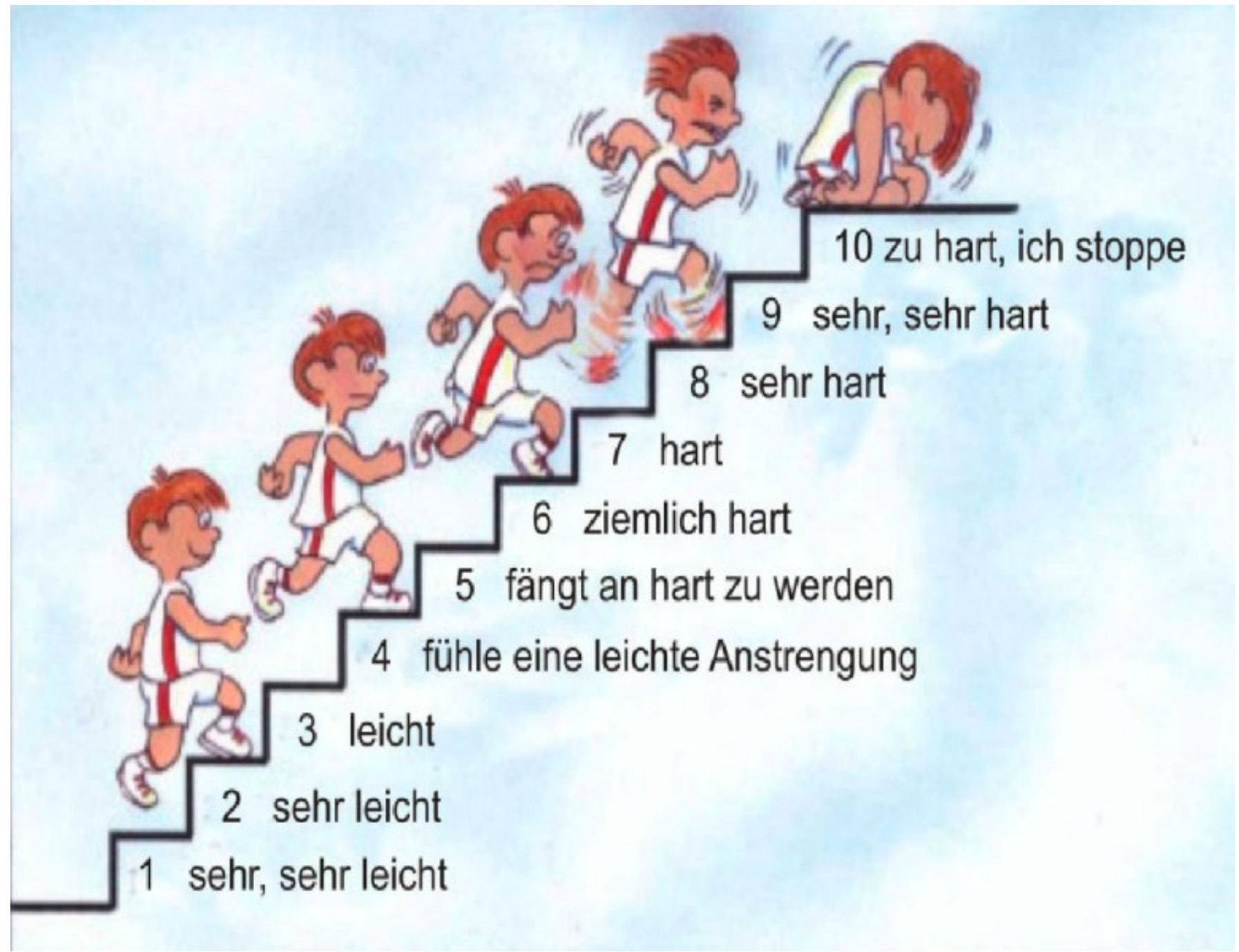

Abb. 1: PCERT Beurteilungsskala der Belastungsintensität. Diese wird in Zahlen, Stufen, Worten und Bildern dargestellt und ist so für alle Altersstufen von Kindern und Jugendlichen geeignet. (PCERT = Pictorial Children's Effort 


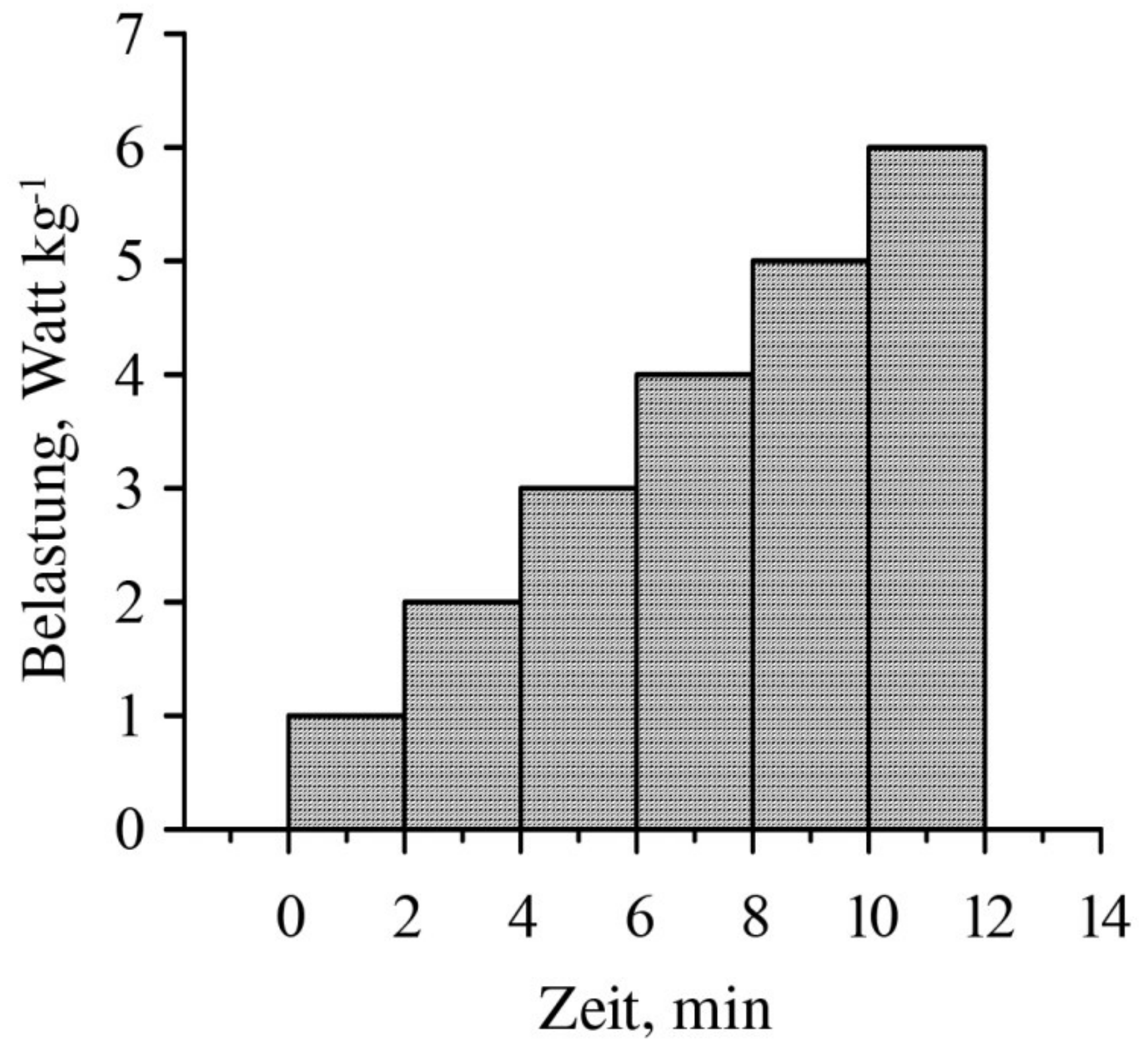

Abb. 2: Giessener Protokoll zur Fahrradergometrie mit Anfangsbelastung von 1 W/kg KG, Inkrement 1 W/kg KG, Stufendauer 2 min. Bei wenig leistungsfähigen Probanden können auch Stufen von 0,5 W/kg KG gewählt werden.

\section{Test-Dauer, Stufendauer, kontinuierliche oder Intervall-Belastung}

Kinder und Jugendliche erreichen schneller ein Steady State im submaximalen Bereich als Erwachsene. Protokolle mit Stufendauer von 2 min gelten daher als gleichwertig zu $3 \mathrm{~min}$. Rampen-Protokolle haben den Vorteil von kleineren Belastungsschritten, die von Kindern gut toleriert werden, jedoch den Nachteil, dass - gerade im submaximalen Bereich - kein Steady State erzielt wird. Messwerte sind damit weniger 
eindeutig einer bestimmten Belastungsintensität zuzuordnen [9].

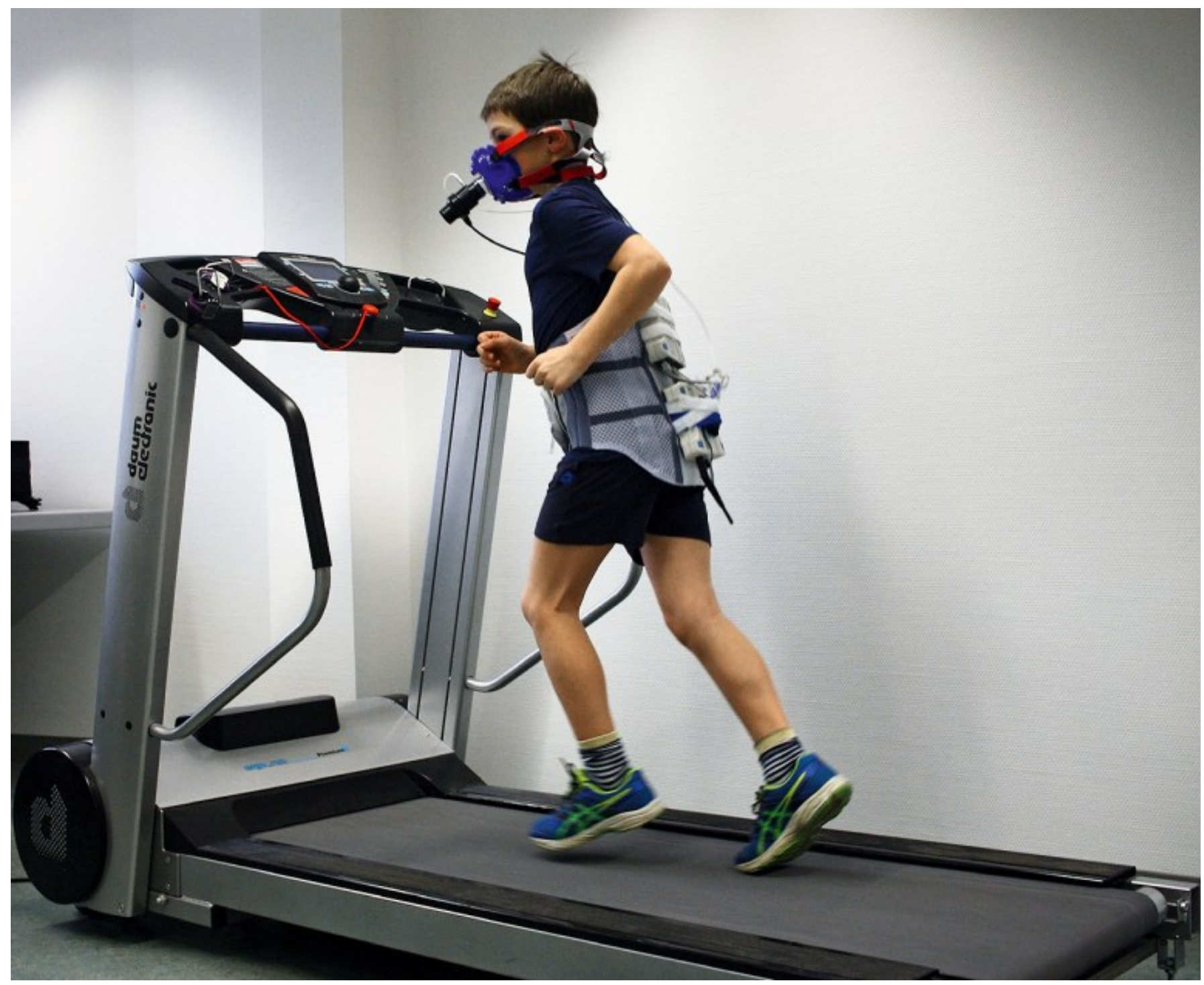

\section{Normwerte und Bezugsgrössen bei Kindern und Jugendlichen}

Die meisten Test-Protokolle verwenden als Bezugsgrössen neben dem chronologischen Alter die Körpermasse/das Körpergewicht (KG), die Körperoberfläche (KOF, m2) oder den Body Mass Index (BMI, kg $\mathrm{KG} / \mathrm{m} 2$ ). Die Normwerte beziehen sich fast ausschliesslich auf das chronologische Alter. Das biologische Alter ist als Bezugsgrösse ausserhalb von Studien praktisch nicht gebräuchlich, obwohl es die Entwicklung der Probanden besser reflektiert als das chronologische Alter. Zumindest das Pubertätsstadium nach Tanner [3,4] muss aber bei jeder Untersuchung miterfasst werden.

Herzfrequenz (HR): Die HR in Ruhe sinkt mit dem Wachstum leicht, die interindividuellen Unterschiede und der Einfluss von Training sind aber beträchtlich. Der Anstieg der HR unter Belastung ist wie bei 
Erwachsenen im submaximalen Bereich linear und im Maximalbereich wird ein Plateau erreicht. Die Maximalwerte betragen über das ganze Wachstumsalter durchschnittlich 200 Schläge, wobei auch hier erhebliche interindividuelle Unterschiede mit bis $\mathrm{zu} \pm 10$ Schlägen pro Minute beobachtet werden. Die Erwachsenen-Formel für die maximale HR von 220-Alter wird von vielen Autoren für Kinder und Jugendliche als inadaequat beurteilt [18,19].

Blutdruck (BD): Der BD in Ruhe steigt während dem Wachstum kontinuierlich. Unter Belastung bleibt der diastolische BD konstant oder sinkt leicht ab, während der maximale systolische BD typischerweise etwa 40 mm Hg über dem Ruhe-BD liegt, jedoch bei grösseren Kindern auch höher ansteigen kann. Die Normwerte bei Belastung werden auf das Alter oder auf die KOF bezogen, und die systolischen Maximalwerte betragen beispielsweise bei KOF 0,7-1,09 m2 142-147 mmHg und steigen mit höherer Körperoberfläche von KOF 1,9-2,31 m2 bis 200-206 mm Hg. Knaben erreichen ca. 10-15 mm Hg höhere Werte als Mädchen mit ähnlicher KOF [18,19].

Sauerstoffaufnahme ( $\mathrm{VO}_{2}$ ): Zur Beurteilung der aeroben Leistungsfähigkeit gilt auch im Kinder- und Jugendalter die Bestimmung der höchsten erreichbaren Sauerstoffaufnahme als einer der wichtigsten Parameter. Der Anstieg der Sauerstoffaufnahme unter Belastung erfolgt im submaximalen Bereich linear zur Belastung, im Maximalbereich wird eine Spitze erreicht (« $\mathrm{VO}_{2}$ max?»), wobei die üblichen Kriterien für den $\mathrm{VO}_{2}$ Maximalwert bei Erwachsenen wie beispielsweise einer genügend langen Plateauphase häufig nicht erreicht und deshalb bei Kindern und Jugendlichen der Begriff $\mathrm{VO}_{2}$ peak adaequat ist. Die PeakWerte sind abhängig von der Körpermasse bzw. dem Körpergewicht, noch genauer von der fettfreien Körpermasse. Die Absolutwerte steigen damit mit dem Wachstum an, die relativen Werte bezogen auf die Körpermasse $\left(\mathrm{VO}_{2}\right.$ in $\mathrm{ml} / \mathrm{kg} \mathrm{KG}$ ) bleiben jedoch über die ganze Wachstumsphase in etwa gleich (Knaben) bzw. sinken nach der Pubertät wegen dem höheren Fettanteil am Körpergewicht leicht ab (Mädchen). Die $\mathrm{VO}_{2}$ max ist weitgehend unabhängig vom verwendeten Protokoll, die auf dem Laufband erreichten Werte liegen 5-10\% höher als auf dem Fahrrad. [20,21,22,23]

Lactat: Die Lactatbestimmung hat einen untergeordneten Stellenwert, da Kinder aufgrund einer geringeren Enzymaktivität und damit niedrigerer anaerober Glycolyse bei gleichzeitig effektiverer Lactatelimination deutlich niedrigere Lactatwerte unter Belastung erreichen als Erwachsene oder auch postpubertäre Jugendliche [24,25]. Allerdings ist die Bandbreite hoch und Kinder mit häufigen anaeroben Belastungen in ihrer Sportart können deutlich höhere maximale Lactatwerte erreichen als ihre Alterskollegen ohne solche Belastungen. Die Bewertung des Lactatanstiegs ist umstritten. Einzelne Autoren gehen von einem relevanten Anstieg bei Werten im Kapillarblut von $+1 \mathrm{mmol} / \mathrm{l}$ über dem Ruhewert aus, während andere den maximalen Lactat Steady State bestimmen. Dieses Vorgehen macht aber mehrere Tests notwendig und wird ausserhalb von wissenschaftlichen Studien kaum angewandt. [26] Maximalleistung: Mädchen erreichen durchschnittliche Maximalwerte von 2,5-3,5 W/kg KG, Knaben von 3,0-3,5 W/kg KG. Die Absolutwerte sind altersabhängig, steigen mit zunehmendem Alter an und liegen ab der Pubertät bei Mädchen etwas tiefer als bei Knaben. [23]

\section{Typische Fragestellungen und ihre Untersuchung (Beispiele)}

Für alle Fragestellungen gibt es verschiedene, oft gleichwertige Untersuchungsmöglichkeiten. Nachfolgend werden exemplarisch zwei bewährte Untersuchungsprotokolle beschrieben.

A. Beispiel-Protokoll für die Abklärung von Dyspnoe und/oder Atemwegsnebengeräuschen bei 
körperlicher Anstrengung. Es dient in erster Linie der Provokation von Atemwegssymptomen (Exercise Induced Bronchoconstriction EIB, Vocal Cord Dysfunction VCD, Intermittent Laryngeal Obstruction ILO) und erst danach der Beurteilung der Leistungsfähigkeit ( $\mathrm{VO}_{2} \max$, Wmax).

Initial Spirometrie in Ruhe, danach ohne Aufwärmen Ergospirometrie. Auf dem Laufband mit Anfangsgeschwindigkeit $>7 \mathrm{~km} / \mathrm{h}$, Stufenhöhe $1.2 \mathrm{~km} / \mathrm{h}$, Stufendauer $2 \mathrm{~min}$, Steigung $1 \%$, Belastungssteigerung bis eine Herzfrequenz $>170$ erreicht ist, entsprechend ca. 80-90\% der maximal erwarteten HR von 200. Um möglichst rasch den Ziel-Herzfrequenzbereich zu erreichen, kann das StufenInkrement bis $1.8 \mathrm{~km} / \mathrm{h}$ erhöht und die Stufendauer verkürzt werden. Auf dem Fahrradergometer Anfangsbelastung $1 \mathrm{~W} / \mathrm{kg} \mathrm{KG}$, Steigerung um $1 \mathrm{~W} / \mathrm{kg} \mathrm{KG}$ pro weitere Minute. Bei HR $>170$ fortgesetzte Belastung für 6 (4-8) min, mit Belastungssteigerung bis zur Ausbelastung. Nach Belastungsende Spirometrie nach 1-2/5/10/15/20 min sowie nach Broncholyse mit zwei Hüben eines kurzwirksamen Bronchodilatators, wobei Salbutamol nach Dopingvorschriften unbedenklich ist. Die Belastungszeit von 6 min bei 80-90\% von HR 200 gilt als wirksamste Provokationsbelastung. [27]

Als anstrengungsinduzierte pulmonale Obstruktion (EIB) gilt bei Kindern und Jugendlichen eine Reduktion von FEV1, wobei eine Reduktion um $>10 \%$ als abnorm und $>15 \%$ als klar pathologisch gilt. Je jünger ein Kind, desto früher nach Leistungsabbruch wird der Tiefstwert des pulmonalen Flusses/der Höchstwert der Obstruktion erreicht, oft bereits nach 3 min [28]. Laufbandprotokolle weisen eine Sensitivität für die Diagnose einer EIB von ca. 60\% aus, Fahrradprotokolle noch etwas weniger. Wohl aus diesem Grund gibt es mehrere Tests mit Surrogatmarkern, wobei deren Sensitivität nicht höher ist. Wichtig ist es daher, einen möglichst sportartspezifischen Test zu wählen und die geklagten Symptome effektiv zu provozieren. Als notwendige Umgebungsfaktoren gelten eine Temperatur $<25^{\circ} \mathrm{C}$ und eine relative Luftfeuchtigkeit $<50-60 \%$, je tiefer, umso intensiver fällt der Provokationsreiz aus. Auch bei Kindern bleibt nach wie vor unklar, ob die Abkühlung oder die Austrocknung der Bronchialschleimhaut durch die vermehrte Ventilation bei Anstrengung der wichtigere Faktor für die nachfolgende Bronchokonstriktion ist. Der kritischste Punkt ist, die Belastung so zu steuern, dass in den letzten 2 min des Tests die maximal mögliche Ausbelastung erreicht wird und gleichzeitig die geklagten Symptome zuverlässig provoziert werden. Dies ermöglicht es dann, zwischen EIB, ILO/VCD und dem Erreichen der Leistungsgrenze ohne Pathologie als Ursache der Symptome zu unterscheiden und im gleichen $\mathrm{Test}^{\mathrm{VO}_{2}}$ peak und die maximale Herzfrequenz zu bestimmen. Die gleichzeitige Verwendung eines EKG erlaubt zudem zumindest auf dem Fahrradergometer oder in Laufpausen, allfällige Rhythmusstörungen oder eine kardiale Ischaemie zu erkennen.

B. Beispiel-Protokolle für die Abklärung von Erschöpfung («Fatigue»), Übertraining, zur Trainingssteuerung und zur Verlaufsbeurteilung bei chronischen Krankheiten: Stufenprotokoll auf dem Laufband Steigung 1\%, Anfangsgeschwindigkeit 6-7,8 km/h, Inkrement 1,2 km/h, Stufendauer 2(-3)min. Rampenprotokolle auf dem Laufband, Steigung 1\%, Anfangsgeschwindigkeit $6 \mathrm{~km} / \mathrm{h}$, Inkrement 0,6 km/h/min, auf dem Fahrradergometer Anfangsbelastung $1 \mathrm{~W} / \mathrm{kg} \mathrm{KG}$, Inkrement 0,5 W/kg KG/min. Gebräuchlich für jüngere Kinder ist auch ein Rampenprotokoll mit Initialbelastung von $10 \mathrm{~W}$ und Inkrement von $10 \mathrm{~W} / \mathrm{min}$ [8]. Für die Abklärung von medizinischen Fragestellungen kann die Messung der Atmungsparameter unter Belastung (Ergospirometrie) wichtige zusätzliche Messwerte liefern. 


\section{Korrespondenzadresse}

Dr. med. Christoph Stüssi

Facharzt für Kinder- und Jugendmedizin

Sportmedizin

SportClinic Zürich/sihldoc4kids

Giesshübelstr. 15

8045 Zürich

+41(0)442878650/4814646

cstuessi@sportclinic.ch

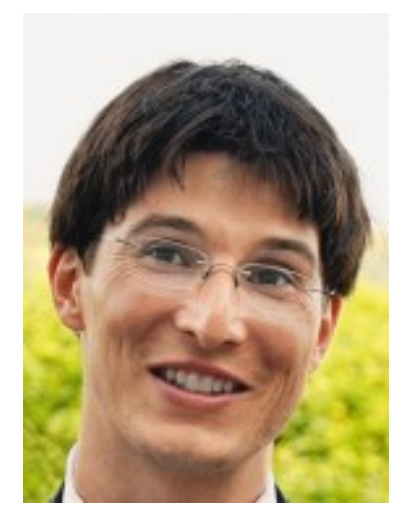

Dr. med. univ. Bernhard Rinderer

Facharzt für Kinder- und Jugendmedizin,

Oberarzt Sportmedizin

Kantonsspital Münsterlingen/Klinik für

Kinder- und Jugendliche/

Sportpraxis am See

Spitalcampus 1

8596 Münsterlingen

+41 (0)716861829

bernhard.rinderer@stgag.ch

\section{Referenzen}

1. Prader A, Largo RH, Molinari L, Issler C: Physical Growth of Swiss Children from Birth to 20 Years of Age (First Zurich Longitudinal Study of Growth and Development). Helvetica Paediatrica Acta 1989;Suppl 52.

2. WHO reconstruction of National Center for Health Statistics NCHS 1977, De Onis M et al.: Länge/Grösse, Gewicht und BMI von 5-18 Jahren aus WHO Growth Charts, Bulletin of the WHO 2007;85:660-667.

3. Marshall WA, Tanner JM: Variation in Pattern of Pubertal Changes in Girls. Arch Dis Childh 1969;44:291-303.

4. Marshall WA, Tanner JM: Variation in Pattern of Pubertal Changes in Boys. Arch Dis Childh 
1970;45:13-23.

5. Nixon PA, Orenstein DM, Kelsey SF et al.: The Prognostic Value of Exercise Testing in Patients with Cystic Fibrosis. N Engl J Med 1992;327:1785-1788.

6. Hebestreit H, Arets HG, Aurora P: Statement on Exercise Testing in Cystic Fibrosis. Respiration 2015;90:332-351.

7. McBride MG, Paridon SM: Congenital and Acquired Heart Disease. 12:141-156. In: Rowland T.W. (Hrsg) Cardiopulmonary Exercise Testing in Children and Adolescents. Champaign, IL: Human Kinetics 2018.

8. Klimt F: Sportmedizin im Kindes- und Jugendalter. Thieme Stuttgart 1992.

9. Myers J.N.: Ramp Exercise Protocols for Clinical and Cardiopulmonary Exercise Testing. Sports Med 2000;30(1):23-29.

10. Myers JN: Essentials of cardiopulmonary exercise testing. Champaign, IL: Human Kinetics 1996.

11. Stephens P, Paridon SM: Exercise testing in pediatrics. Pediatr Clin North Am 2004;51(6):1569-1587.

12. Robertson RJ, Goss FL, Boer NF, et al: Children's OMNI Scale of Perceived Exertion: Mixed Gender and Race Validation. Med Sci Sports Exerc 2000;32(2):452-458.

13. Daley AJ, Copeland R, Wright N, et al: The pictorial children's effort rating table (PCERT). BMC Public Health 2005;5:113.

14. Haile L, Gallagher M, Robertson R.J: Perceived exertion laboratory manual. Springer, New York 2015.

15. Maier Th, Gross M, Trösch S, Steiner T, Müller B, Bourban P, et al: Swiss Olympic Manual Leistungsdiagnostik. Bundesamt für Sport 2015.

https://www.swissolympic.ch/dam/jcr:b15b191a-eb0d-46e8-b9c0-417b887a440d/Leistungsdiagnostik_ Manual_160201_DE.pdf) 5.1.2019.

16. Nowacki PE: Unterschiede und Entwicklungen der maximalen biologischen Leistungsfähigkeit sportund nichtsporttreibender Kinder und Jugendlicher. In: Rieckert H (Hrsg.): Sportmedizin Kursbestimmung. Kongressband 30. Deutscher Sportärztekongress Kiel 1986. Springer 1987:75-84.

17. Selvadurai HC, Cooper P, Meyers N, et al: Validation of Shuttle Tests in Children with Cystic Fibrosis. Pediatric Pulmonology 2003;35:133-138.

18. Rowland T: Cardiovascular function, 11:152-154. In: Armstrong N, van Mechelen W (Hrsg): «Oxford Textbook of Children's Sport and Exercise Medicine». Oxford University Press, Oxford 2017.

19. «Cardiovascular function» In: Armstrong N., van Mechelen W. (Hrsg.) «Oxford Textbook of Children’s Sport and Exercise Medicine», chapter 11, 152-154).

20. Armstrong N, McManus AM: Aerobic fitness 12:161-172. In: Armstrong N, van Mechelen W (Hrsg): Oxford Textbook of Children's Sport and Exercise Medicine. Oxford University Press, Oxford 2017.

21. Armstrong N, Welsman JR, Kirby BJ: Peak Oxygen Uptake and Maturation in 12-yr Olds. Med Sci Sports Exerc 1998;30:165-169

22. «Aerobic fitness» In: Armstrong N., van Mechelen W. (Hrsg.) «Oxford Textbook of Children’s Sport and Exercise Medicine», chapter 12, 161-172.

23. Lawrenz W, Hebestreit H: Ergometrie im Kindes- und Jugendalter 72-84. In: Hebestreit H, Ferrari R, Meyer-Holz J, et al (Hrsg): Kinder- und Jugendsportmedizin Thieme Stuttgart 2002.

24. Eriksson BO, Gollnick PD, Saltin B: Muscle metabolism and enzyme activities after training in boys 11-13 years old. Acta Physiol Scand 1973;4:485-497.

25. Fournier M, Ricci J, Taylor AW, et al : Skeletal muscle adaptation in adolescent boys : sprint and endurance training and detraining. Med Sci Sports Exerc 14;6:453-456.

26. Armstrong N, McManus AM: Aerobic fitness 12:173-174. In: Armstrong N, van Mechelen W (Hrsg): Oxford Textbook of Children's Sport and Exercise Medicine. Oxford University Press, Oxford 2017.

27. Crapo RO, Casaburi R, Coates AL, et al: Guidelines for Methacholine and Exercise Challenge Testing 1999. Am J Respir Crit Care Med 2000;161(1):309-329.

28. Vilozni D, Szeinberg A, Barak A, et al: The Relation between Age and Time to Maximal 
Bronchoconstriction following Exercise in Children. Respir Med 2009;103(10):1456-1460.

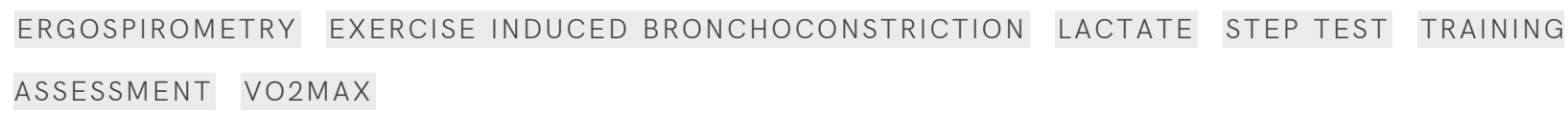

